\title{
Application of Weighted cetroid location algorithm in Forest Fire Monitring
}

\author{
Chunliang Zhou \\ Engineering College \\ Ninbo Dahongying University \\ Ningbo,china \\ Guest130@163.com \\ Weiqing Qu \\ Engineering College \\ Ninbo Dahongying University \\ Ningbo,china \\ 852721090@qq.com
}

\author{
Zhengqiu Lu \\ Engineering College \\ Ninbo Dahongying University \\ Ningbo,china \\ 459246322@qq.com \\ Qi Zhang \\ Engineering College \\ Ninbo Dahongying University \\ Ningbo,china \\ 449101398@qq.com
}

\begin{abstract}
In the internet of things for forest data monitoring, the horizontal and vertical coordinates of a fire point could be calculated by the weight of the sensor node. By introducing the concept of weighted centroid localization, the fire point can be quickly positioned. The algorithm provides a strong basis for fire point positioning in forest fire monitoring by comparison experiment, which can reduce forest fires loss.
\end{abstract}

Keywords-Internet of things; data monitoring; weighted centroid localization

\section{InTRODUCTION (HEADING 1)}

In 1999, the MIT Auto ID Center proposed the earlier international definition of the "Internet of Things"--- On the basis of the computer Internet, to construct a network covering everything in the world in order to achieve the automatic identification of goods and Internet sharing of information by using radio frequency identification (RFID), wireless data communications technology and so on. In 2005 the International Telecommunication Union (ITU) released ITU Internet Report 2005: Internet of Things officially and presented the concept of the "Internet of Things"--- an extension of the Internet applications, and RFID, sensor technology, nanotechnology, smart embedded technology will be the four core technologies of Internet of Things.

Domestic definition of Internet of Things is: a huge network formed by combining a variety of sensing devices, such as radio frequency identification (RFID) devices, infrared sensors, global positioning systems, laser scanners, other various devices, and also the Internet. Its purpose is to make all of the items are connected to the network, so the system can automatically and in real-time object recognition, localization, tracking, monitoring, and trigger the corresponding events.

The Internet of Things are extremely versatile, and has been applied to the monitoring of the various natural disasters, health care, power systems, intelligent transportation, intelligent community management, military, aviation and aerospace field. As another revolution in the information industry, the Internet of Things will also be a decisive force in the construction of digital forestry. At present, Internet of Things have been studied in field of fire in the forest, trees management, protection of rare wild animals, timber tracking management, seedlings, flower cultivation fronts. The paper studied sensor positioning algorithm for detection of forest fires based on the monitoring of forestry data networking by Internet of Things system, which could accelerate fire positioning, and maximize the prevention and reduction of forest fires and the resulting loss. Type Style and Fonts

Wherever Times is specified, Times Roman or Times New Roman may be used. If neither is available on your word processor, please use the font closest in appearance to Times. Avoid using bit-mapped fonts if possible. True-Type 1 or Open Type fonts are preferred. Please embed symbol fonts, as well, for math, etc.

\section{INTERNET OF THINGS ARCHITECTURE BASED ON FORESTRY DATA MONITORING}

The perceived link of Internet of Things has a strong heterogeneity, and was divided into 4 levels based on open, layered, scalable network framework structure in order to further achieve the information interconnection, interoperability and interoperability of forestry data monitoring: the sensor network layer (sensor placement, radio frequency identification systems, etc.) $\rightarrow$ network access layer (gateways, bridges, routers and other networkconnected devices) $\rightarrow$ middleware layer (data storage server, monitoring data) $\rightarrow$ application layer (results are presented to the system administrator, and feedback to the bottom of Internet of Things).

(1) In the sensor network layer, the sensors collect various types of monitoring signals, and transmit them to the next level of cyberspace through the convergence of wireless sensor node.

(2) The network access layer deal with data fusion or pretreatment. The main network was interconnected by bridges, gateways, routers, switches and other network equipments, and then processed data received from the 
sensor network layer to the data server in the middleware layer.

(3)The middleware layer store data from sensor network layer connected by network equipment, deal with data classification, data analysis algorithms and models, and provide effective protection for monitoring data results oriented the application layer.

(4)The application layer presents results from middleware layer algorithms and models to the administrator at the application layer, and on the other hand, sends information back to the ground floor of Internet of Things according to the application requirements to achieve the corresponding control.

\section{LOCATION ALGORITHM IMPLEMENTATION}

\section{A. Algorithm proposed}

In the Monitoring of forest fires, it is necessary to detect wind speed, temperature, humidity and other parameters around the points of forest, and identify fire locations in case of fire accurately. The forest, covering a large harsh environment, with many unattended area, requires collaboration of a large number of nodes to complete the monitoring tasks. So the advantage of wireless sensor network for monitoring is apparent. The location of the fire when the fire broke out in the forest fire monitoring system is vital essential, which is also one basic functions of the sensor network.

At present, there are many ways to accurately position the location of information, for example, Global Positioning System (GPS) positioning. But the user nodes are usually of high energy consumption, large volume, high cost, and it also need a fixed infrastructure. Therefore, in the case of GPS applications are limited, sensor networks with small size, low power consumption, low-cost sensor networks can well solve the positioning problems. The typical wireless sensor network positioning technology consist of methods based on the received signal strength indication (RSSI), time of arrival (TOA), time difference of arrival (TDOA), angle of arrival (AOA). However, these methods need additional hardware supports. Although RSSI positioning technology does not need additional hardware support, the RSSI-based positioning results are often unstable, so its application is restricted. Here, the weighted centroid location algorithm is applied to the monitoring of forest fires. The algorithm is based on centroid localization algorithm, it can determine the different influence of the perception of nodes for target detection by Markov chain forecast, and reasonably divide target location to identify the trajectory of targets, position the sensors monitoring fire accurately, and determine the fire took place.

\section{$B$ Description of the algorithm}

Nirupama Bulusu from University of Southern California proposed the Centroid algorithm for sensor positioning, the main idea of the algorithm is: the sensor sensing node detect the target from time to time, when the target appears in the sensing region of perception nodes, the target location was determined as the polygon centroid composed by the perception nodes, the average of all vertex coordinates of the polygon is the coordinate of the centroid node. When the system has been able to detect location information of perception nodes, the current position of the target can be estimated according to the vertex coordinates of polygon consisting of these perceptions nodes.

The weighted centroid localization algorithm is an improvement of location centroid algorithm, it can reflect influence of the perception node on target location by a weighting factor, and is a localization algorithm based on network connectivity. The algorithm is as follows:

Assume the sensor network node set is $S=\{\mathrm{s} 1 、 \mathrm{~s}$ $2 、 \cdots$, s $N\}$, Each node can sense the scope of Ai, the sum sense scope of $\mathrm{N}$ nodes is $\mathrm{Ar}=\sum \mathrm{Ai}$. When two nodes do not coincide, and the perception of the region do not intersect, every moment there is only one node can sense the target and the target position in the next moment correlate with the current location.

If $\mathrm{Xn} \log$ the number of perception node regions target appears in the network at time $n$, then $\left\{X_{n}, n=1,2 \cdots\right\}$ is a Markov chain, and the state set is $\{1,2, \ldots, N\}, X_{t}=i$ represents the target appears in the perception region of $\mathrm{Ai}$ at time t. Sensing nodes can sense the target appeared in the corresponding perception area at every moment, therefore, when Markov chain enter the next state, it will lead to a change of perception node detection value.

Assume, when Markov chain enter the state j, independent of the previous state and change the detection value of $s_{j}$ with a probability of $p(j \mid i)$. When $s j \in n r_{s i}, \sum s j \in n b r_{s i} p(j \mid i)$ $=1$, when $s_{j} \in n b r_{s i}, p(j \mid i)=0$. The detection value can be observed through the detection the target by nodes.

$Y_{i t} \in\{0,1\}$ represents the detected value of the node $i$ at time $\mathrm{t}$, to detect the target value is 1 , not to detect the target value is 0 . Therefore, $\mathrm{Y}_{\mathrm{t}}=\left(\mathrm{Y}_{1 \mathrm{t}}, \ldots, \mathrm{Y}_{\mathrm{Nt}}\right)$ means all the detection results of all sense nodes at time t. $\mathrm{Y}_{\mathrm{t}}=(\mathrm{Y}$ $\left.1, \cdots, Y_{T}\right)$ is the result of all the detection before time $t . X$ $1, \mathrm{~T}=\left(\mathrm{X}_{1}, \cdots, \mathrm{X}_{\mathrm{T}}\right)$ is the state set of targets before time t. According to the description above, the joint distribution function can be drawn:

$$
p\left(x_{i, T}, Y_{1, T}\right)=p\left(x_{1}\right) \prod_{t=2}^{T} p\left(x_{t} \mid x_{t-1}\right) \prod_{t=2}^{T} p\left(Y_{t} \mid X_{t}\right)(1)
$$

$\delta$ ( i) represents the maximum possibility at time i $(\mathrm{i}=$ $1, \cdots, \mathrm{N}, \mathrm{t}=1, \cdots, \mathrm{T})$ :

$$
\delta(i)=\max p\left(x_{1 .} . x_{t-1}, x_{t}=i, y_{1, t}\right)(2)
$$

$\mathrm{P}($ zit $\mid z j t-1)$ represents probability of the target moving from sensing area of $\mathrm{j}$ node to $\mathrm{i}$ node. Since probability is non- negative, and the process must be transferred to one state, so, when $\mathrm{j} \notin \mathrm{nbr}, \mathrm{P}\left(\mathrm{z}_{\mathrm{it}} \mid \mathrm{z}_{\mathrm{jt}-1}\right)=0$. suppose that possibility of Si is $\eta \mathrm{I}$, then $\mathrm{P}\left(\mathrm{y}_{\mathrm{it}}=1 \mid \mathrm{z}_{\mathrm{it}}\right)=\eta \mathrm{I}$ and $\mathrm{P}\left(\mathrm{y}_{\mathrm{it}}=0 \mid\right.$ $\left.\mathrm{Z}_{\mathrm{it}}\right)=1-\eta \mathrm{I}$, and the formula (3) can be deriven: 


$$
\delta t(i)=\left[\max p\left(z_{t}^{i} \mid z_{t-1}^{j}\right) \delta_{t-1}(j)\right] \eta^{y_{t}}\left(1-\eta_{i}\right)^{1-y_{i}}
$$

By means of Baum-Welch method, the Markov model parameters $\pi, \eta, P t=[p i j]$ can be calculated through historical information perceived by the sense nodes of the network. Through the node's own information, weighted value $\delta \mathrm{t}(\mathrm{i})$ of each node can be calculated, where: $\delta_{\mathrm{t}}=$ $\pi \eta^{\mathrm{yi}(1-n \mathrm{i})}$.

At time t, coordinates with $\delta \mathrm{t}$ value greater than 0 are as follows : $\left(\mathrm{x}_{1}, \mathrm{y}_{1}\right) 、\left(\mathrm{x}_{2}, \mathrm{y}_{2}\right) 、 \cdots,\left(\mathrm{x}_{\mathrm{n}}, \mathrm{y}_{\mathrm{n}}\right)$, and weighted value of each corresponding sensor nodes are:

With the weighted value, the coordinate of the target can be drawn:

$$
x=\sum_{i=1}^{n} w_{i} x_{i}, y=\sum_{i=1}^{n} w_{i} y_{i}(5)
$$

By the algorithm description above, this kind of node weights can be deduced according to the sensing area of sensor nodes, the final location of each sensor nodes are approximately located.

\section{USING THE TEMPLATE ALGORITHM ACHIEVING AND RESULT}

In the experiment, the network nodes are randomly deployed in a rectangular area. Assume that node coverage and node communication radius can be adjusted, the location of the nodes in the network is known, and experimental results of randomized 30 times are shown in Figure 1(The square means the average weighed location, and the circle means the centroid weighted localization). When node density is very low, the average error of centroid positioning is great, but the weighted positioning error is small. As the node density increases, when it is near to 10 percent, the average error of each localization algorithm is close.

\section{CONCLUSION}

This paper introduced the status of the Internet of Things and Internet of Things application architecture system. Fire monitoring sensors deployed in the forest receive data through the network access layer transmit to the data memory and carry out fire point positioning. In this paper, the centroid localization algorithm in the Internet of Things technology is introduced, and the shortcoming of positioning error in the algorithm is also improved. Based on Weighted centroid localization algorithm, the weighted value of sensor nodes was calculated by the perception area of the sensor nodes, thus rapid positioning of the ignition point was completed and the losses caused by forest fires were reduces. By simulation tests, the average location error of centroid localization algorithm error rate in the case of smaller node density is relatively high, but the average location error of the weighted centroid algorithm change gently. Overall, how to reduce the error rate remains to be further improved.

\section{ACKNOWLEDGMENT}

The work was supported by the Information College. Thanks for the Mr Huang and my colleague . Thank you for my student to handle the issues.

\section{REFERENCES}

[1] Cooper P .International Telecommunication Union, Internet Reports 2005: The Internet of things. Geneva, 2005.

[2] Philip B. Commission of the European communities. Internet of things-an action plan for Europe, 2007.

[3] European Research Projects on the Internet of Things Strategic Research Agenda. Internet of things-strategic research roadmap, 2009.

[4] Wolf W. Cyber-physical Systems Computer, 2009, 42(3):88-89.

[5] LEE E A. Cyber Physical Systems: design challenge.11th IEEE, 2008.

[6] Amadeus C. Symposium on Object Oriented Real-Time Distributed Computing.2008:363-369.

[7] Kevin Ashton. International Telecommunication Union UIT Internet Reports, 2005.

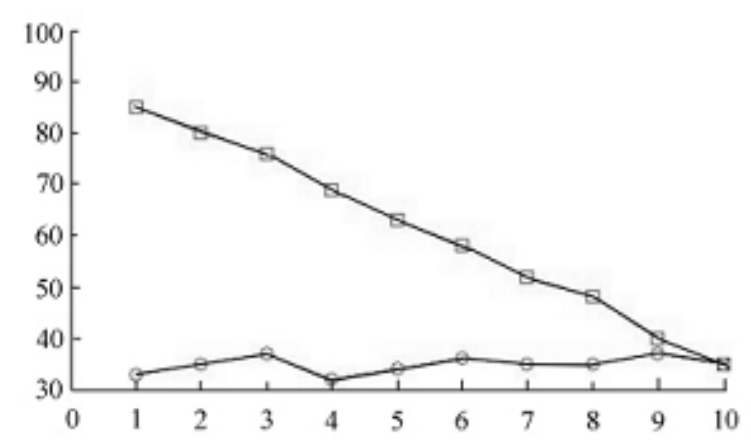

Figure 1. the simulation on the matlab 